\title{
Index
}

A new response to a changing neighbourhood (2011) 40, 47, 70

Action Plan(s) 49, 51, 87

advocacy coalition framework (ACF) 11,181

Agenda for Migration 2015 (EU) 147

Alliot-Marie, Michèle 55, 58, 62, 67, 69

Allison, Graham T. 7

Allison, Roy 75, 96, 97, 129, 130

ambiguous policy change, see: constructive ambiguity

analogical reasoning 5

analytical framework of EU foreign policy change 15-22, 23-44

Ashton, Catherine 56, 57, 58, 69, 70, 72, 83, 97, 108, 109, 119

Association Agreement (AA) 40, 48, 49, 51, 75, 85, 86, 129

asylum crisis, see: European asylum crisis

Baltic Sea Strategy 62

Barroso, José Manuel on Arab uprisings 56, 58, 70, 119

on Euromaidan 84, 96

Bauer, Michael W. 35

Baumgartner, Frank R. 11, 12, 26, 35, 36, 39, 120

Ben Ali, Zine El Abidine 1, 46, 58, 67, 68

Berlusconi, Silvio 55, 68

Boin, Arjen 11, 39, 40, 72, 112, 120 , 183, 189

Börzel, Tanja 2, 9, 152, 177

Bouazizi, Mohamed 40, 46
Budapest Memorandum 76

Buzan, Barry 6

Capoccia, Giovanni 120, 128, 179

Carlsnaes, Walter 7, 8, 27

Cashore, Benjamin 12, 17, 18, 35-7, $181,182,184$

causality 22,25

causal filters 38 contextual 26, 32, 37

Civil Society Facility 71, 72

Clinton, Hilary 25, 32

Common Foreign and Security Policy (CFSP) 38, 51, 59, 60, 86, 87, $92,159,176,182$

Common Security and Defence Policy (CSDP) 159, 160, 166, 176, 184

conditionality 50, 53, 59-61, 63, 66, $71,77,87,88,91,110,116$, 186

conjunctural context 21, 33, 34, 47, 67, 99, 118-20, 135, 137, 152, 168-9, 171, 180

constructive ambiguity 17-19, 34, 44, 101, 104, 106, 112, 118-123, 183, 185-90

definition 114-17

examples 186-188

contingency (see also: temporal contingency) 21, 24, 26, 27, 29, 40, 118, 135, 146, 149, 162, 165,180

definition 31-34

and the Arab uprisings 66-70

and the Ukraine conflict 94-9

Court of Justice of the European Union (CJEU) 31 


\section{Crimea 2}

annexation 2, 75, 77, 80, 83, 95-6, 99, 103, 114, 130-1, 137, 158, $161-2,165,169,171$

‘little green men' 130

Crisis

as catalysts of change $5-6,12-15$

asylum 142-158

framing $6,23,42,56,58,65,73$, 84, 91, 92, 109, 126, 134, 170, 189

in IR theory 5-6, 176

in security and defence 158-174

migration see: European asylum crisis

of disinformation 125-142

refugee see: European asylum crisis

Critical juncture(s) 5, 14, 16, 18-22, 38,39

definition and features 27-31, 42-4 perceptions of 40

necessary conditions 30

Day of Rage 28 January 2011 (Egypt)

Decision-making

authority 52, 117

process of EU foreign policy change 24

Deep and Comprehensive Free Trade Agreement (DCFTA) 75, 78, 92, 100, 102

diachronic causality 25

Dingley, James 18, 101, 115, 188

directionality (of policy change) 17 , 24, 36, 44, 59-61, 87-90, 100, 108, 109, 111, 118, 120-1, 136, $150-1,166-7,182-3$

disinformation

definition 126

as a Russian strategy $125-142$

Dublin System 142, 146-8, 150-5, 157

East StratCom Task Force

legal basis 138, 139

overview 138, 139, 140

Eastern Partnership (EaP) 39, 52, 76, $79,136,140$

countries 78

Russian perception 16, 84, 103, 126
Egypt

demonstrations 46-7

president of (see also: Mubarak, Hosni)

spread of Arab uprisings to 48, 67, 68-9

Enlargement

fatigue 50

policy 74, 76, 78, 100, 102, 109, 110,159

Epistemology 37

EU strategic communications 125, 127-8, 131, 133, 134, 137-142

Euro-Mediterranean Partnership (EMP) 48, 52, 55, 64, 73, 179

Euromaidan (see also: Maidan) 83, 84, 95, 99

European Army 161, 164, 170

European asylum crisis 142-158

European Defence Agency 41, 164, 171-2

European Defence Fund (EDF) 166, $167,171,172-3$

European Neighbourhood Instrument (ENI) 37, 88-9, 93-4, 102, 110

European Neighbourhood Policy (ENP)

and the European Commission 49

funding, see: European

Neighbourhood Instrument legal basis $30-1,38,51-2$ summary of $16-17$

European Union Global Strategy

(EUGS) 161-3, 168

European Union Institute for Security

Studies (EUISS) 128, 161

evidence 41-2

account evidence 41

and historical process tracing 41-2

false paradigm change, see: faux-

paradigmatic policy change

faux-paradigmatic policy change

17-18, 184-5

Fioretos, Karl Orfeo 15, 25, 180

foreign policy analysis (FPA) 4, 6-8, $12,23,181,188$

foreign policy change

along Guttman scale 7, 12, 13, 107, 181,188 
cyclical models of 7

in EU focused literature 8-10

input-output models of 6

Frattini, Franco 53, 65-6, 69-70

Freedman, Lawrence 75

Frontex 65, 69, 142-3, 145, 149, 150, 153, 155-7

Füle, Štefan 57, 70, 84, 108, 119

Gafsa revolts 2008 (Tunisia) 47

Gilpin, Robert 5

Glazyev, Sergei 78, 79

Goldmann, Kjell 5, 7

Gourevitch, Peter 26

Gustavsson, Jakob 7, 12, 35, 188

Guttman scale of change 7, 12, 13, 107, 181, 188

Hahn, Johannes 80, 85, 99-100, 109-10

Hall, Peter 5, 11-13, 17, 25-6, 29-30, $34,36-7,70,121$

typology of change 106-9, 111, 114, 181

Helsinki Act 76

Hermann, Charles F. 6, 7, 12, 35, 107, 181, 188

historical institutionalism (HI)

and EU foreign policy change 27-37

and policy change 25-6

as an approach to studying politics $3-4$

second wave 4, 26, 27, 43, 179

historical method (see: historical process tracing)

historical process tracing 24, 36-41, 124-5

and evidence 41

and interviews 42

and structured episodes 32

Holsti, Kalevi J. 6, 181, 188 markers of change 12-14

Howlett, Michael 12, 17, 18, 29, $35-7,181-2,184$

hybrid conflict 129, 133, 135

Ikenberry, G. John 5

Immergut, Ellen 25-7

information operations, see: disinformation information warfare, see: disinformation

institutional

as a factor of change $26-7,178$

change $14,21,30,38,121,180$

effects 12, 26-7, 30-31, 37-8, 76

leeway 38, 52, 76

legacy, example of 178

plasticity $70,112,117-18,122$, $128,154,161,178$

lock-in effects 115

institutions

and path dependency 5, 21, 26, 187

and plasticity (see: institutional plasticity)

and policy change $30-1$

and power 52, 117

as dynamic arenas of conflict 25, 30

co-existence 52, 115, 185

constraining $30,52,128,139,145$, 179

definition 3-4

malleability 15, 30-1, 43, 52, 73, $128,145,160,178-9$

overlapping $52,73,78$

interpretative flexibility $38,52,179-80$

Jegen, Maya 18, 115-16, 122, 185-6, 190

Jones, Bryan D. 11, 12, 26, 35-6, 120

Juncker, Jean-Claude 76, 85, 95, 103, 109, 146, 161-3, 165, 170

Juppé, Alain 58, 70, 108

Kelemen, R. Daniel 2, 28-9, 31, 177

key actors

categorising and defining 39-40, 43-4

Khaled Said 46

Kingdon, John W. 10, 30, 120

Knill, Christoph 35

Krebs, Ronald R. 6, 189

Lebow, Richard Ned 32

Libya crisis $41,47,53,62,68-9,12$, 159

liminality 21, 33-4, 67, 73-4, 99, 113, 118-20, 135, 137, 142, 152, 168, 169, 171, 180, 190 
Lithuania 131, 132, 134, 138

little green men 130

Macron, Emmanuel 163, 168

Maidan, see: Euromaidan

Malmström, Cecilia 58

Mare Nostrum 145

McConnell, Allan 11, 29, 39, 72, 112, 120, 183, 189

Mérand, Frédéric 18, 115, 116, 122, 158, 185-6

Merkel, Angela 55, 62-3, 67, 82, 86, 91, 96, 98, 108, 147, 159

MH17 disaster 44, 77, 83, 95, 98, 99, 114

migration crisis, see: European asylum crisis

migration policy EU 142-4

Military Planning and Conduct Capability (MPCC) 171-3, 184-5

minilateralism 52, 82, 99

Mogherini, Federica 80, 131, 134, 138, 146, 158, 163, 170, 173

Mubarak, Hosni 1, 47, 55, 67-9

multiple streams framework (MSF) 10-11

Nisbet, Robert A. see also: directionality 36

objectivity 37,39

ontology 37

outcomes of policy change

most likely 117-21

for Arab uprisings 71

for Ukraine crisis 102

for crisis in security and defence 172

for migration and asylum crisis 155 for rise in disinformation crisis 140 revised typology 111-17

\section{Painful choices: a theory of foreign} policy change (David Welch) 22, 33, 35, 124

Palier, Bruno 18, 115-16, 139, 185 paradigmatic (policy) change 12, 26, 72,114
Partnership for Democracy and Shared Prosperity for the Southern Mediterranean (PfDSP) 57

path dependency 5, 21, 26, 187

perception management, see: disinformation

Permanent European Structured Cooperation (PESCO) 163, 166-7, 169-74

pillar system (EU) 159

plastic

institutions $15,26-7,160,178-80$

plasticity

examples of $31,144,178$

definition 30

Police Day Egypt, 25 January 201146

policy

components, see: policy disaggregation

disaggregation $17,35,37,111,127$, 182

entrepreneurship 38-9, 52, 73, 76, $78-9,99,128,179$

levels 24, 37

policy change

ambiguous, see: constructive ambiguity

an analytical framework of 15-22, 23-44

as accommodating gestures 17 , 112-13, 120, 171, 183, 185

categorising 14, 111-17

directionality of, see: directionality (of policy change)

disaggregating $17,35,37,111,127$, 182

existing theories and

conceptualisations 4-12

faux-paradigmatic 17-18, 184-5

in international relations

in public policy studies

levels 24, 37

orders of 106-7

symbolic $112-14$

typology of 111-17

Policy paradigms, social learning, and the state (Peter Hall)

polysemic policy change, see: constructive ambiguity 
Poroshenko, Petro 82

process tracing (see: historical process tracing)

Prodi, Romano 50

public policy studies 4, 10-12, 17-18, 23-4, 35, 112, 115, 122, 176, 181-3, 185

punctuated e quilibrium 11, 26

Putin, Vladimir 1, 78-79, 82, 95, 130, 158,165

Qhadafi, Muammar 22, 69

rational choice theory 10,25 , 32

reflexive control, see: disinformation refugee crisis, see: asylum crisis

Review of the European

Neighbourhood Policy (ENP): stronger partnerships for a stronger neighbourhood (2015) 40, 69, 93

Rose, Richard 112, 183

Sabatier, Paul A. 11, 35-6

Sakwa, Richard 76, 81, 92

salience $15,33,48,67,70,74,76,99$, 103, 112-13, 119-120, 131, 141,181

Sarkozy, Nicolas 55, 62, 67

Schengen Agreement 142, 144-5, 147, $149,152-3$

Schetyna, Grzegorz 92

Security Union (EU) 162

Sidi Bouzid (Tunisia) 46

Sikorski, Radosław 96

Simferopol (Crimea) 75, 130

Skidmore, David 7

Skocpol, Theda 25, 41

Soifer, David H. 27-8

Steinmeier, Frank-Walter 148, 164

Steinmo, Sven 4, 12, 25-6

strategic communications (see also: East StratCom Task Force)

strategic disinformation, see also: disinformation 127-8, 125-42

structural context 33-4, 47, 50, 119, 149,180
Support to Partnership, Reforms and Inclusive Growth (SPRING) 71-2, 113

symbolic policy change

definition 17, 112-14, 183

examples of 113,183

synchronic causality 25

Tahrir Square (Cairo, Egypt) 47

technocratic logic 38, 49, 52, 73-4, 76, 99, 104, 118, 122

temporal

context 180

contingency $21,24,26,31-34$

registers $21,33-4,135,180$

terrorism 49-50, 65, 74, 102, 142-3, $158,162-3,168$

't Hart, Paul 29, 32, 72, 112, 120, 183,189

Thelen, Kathleen 12, 25-6, 30, 42

time

impact of see: temporal registers, temporal contingency

timescape see: temporal context

Tocci, Nathalie 9, 168-9, 171

Trump, Donald 1, 25-6, 28, 165, 168, 173

Tunisia 1, 40, 41, 46-7, 53, 58, 60, $62,64,67-9,100,151$

Tusk, Donald 133, 149

typology of change

traditional three-level 7, 12-13, 17, 106

suggested new typology 111-17

Union for the Mediterranean (UfM)

$39,52,55,58-9,61-4,66-9$,

73, 79, 101-12, 117, 179, 187

Van Rompuy, Herman 56, 57, 69, 96

Viktor Yanykovych

deal with 76-7, 95

ousting and flight 96

Vilnius U-turn 79-80, 95-6

Vilnius Summit (2013) 79-80, 95-6

Visegrád Group 97, 151, 154

We are all Khaled Said (movement Egypt) 46 
Weimar Triangle 76, 81-2, 86, 92-4, 96, 97, 99, 103-4, 168

Welch, David 7, 22, 33, 35, 113, 120, 124

Westerwelle, Guido 63, 69, 109

Whitman, Richard G. 50, 53, 80, 158

Why nations realign (Kalevi J. Holsti) 6, 12-14, 181, 188 window of opportunity 10, 28, 137, 188

Youngs, Richard 2, 8, 48, 55, 159, 177, 182

Zelikow, Philip 7

Ziblatt, Daniel 32, 34, 38-9, 44 